\title{
Actividades extracurriculares y su relación con el estilo de vida de los estudiantes de enfermería
}

\section{Extracurricular activities and their relationship with the lifestyle of nursing students}

\author{
Arias Ulloa, Raúl; Reyna Solórzano, Mónica Lizbeth; Díaz Parra, \\ Cecilia Elizabeth; Alvarado Martínez, Ruth; Arroyo Hernández, \\ Paula Patricia; Macías Romo, María Karen
}

Raúl Arias Ulloa raul.arias@edu.uaa.mx

Universidad Autónoma de Aguascalientes, México

Mónica Lizbeth Reyna Solórzano

Universidad Autónoma de Aguascalientes, México Cecilia Elizabeth Díaz Parra

Universidad Autónoma de Aguascalientes, México

Ruth Alvarado Martínez

Universidad Autónoma de Aguascalientes, México

Paula Patricia Arroyo Hernández

Universidad Autónoma de Aguascalientes, México María Karen Macías Romo

Universidad Autónoma de Aguascalientes, México

Lux Médica

Universidad Autónoma de Aguascalientes, México

ISSN: 2007-1655

Periodicidad: Cuatrimestral

vol. 17 , núm. 49, 2022

luxmedica.editorial@gmail.com

Recepción: 28 Junio 2021

Aprobación: 09 Diciembre 2021

URL:

https://revistas.uaa.mx/index.php/luxmedica/article/view/3267

DOI:

https://doi.org/10.33064/491m20223267
Resumen: Introducción: Las actividades extracurriculares son aquellas ocupaciones no pertenecientes al currículo escolar, pero que influyen en la persona para desarrollar habilidades y capacidades recreativas que pueden tener impacto en el estilo de vida de los estudiantes de enfermería. Objetivo: Demostrar la relación de las actividades extracurriculares con los estilos de vida de los estudiantes de enfermería de la Universidad Autónoma de Aguascalientes. Métodos: Estudio descriptivo transversal a nivel relacional, realizado a estudiantes de Enfermería con una muestra de 221 estudiantes, obtenida por muestreo probabilístico aleatorio simple sin reemplazo. Se utilizó el cuestionario "FANTASTIC $® "$, con un Alfa de Cronbach de 0.789. Resultados: La edad promedio de la población estudiada fue de 20 años, predominando el sexo femenino, con un $81.9 \%$. El estilo de vida conforme a la autopercepción del estudiante fue bueno, en un 52\%. La mayoría de los estudiantes no realizaban actividades extracurriculares, con un $55.2 \%$. No se encontró asociación estadísticamente significativa entre el estilo de vida y las actividades extracurriculares ( $c 2=0.603, \mathrm{p}=.896)$. Conclusión: Las actividades extracurriculares no siempre serán significativas en el estilo de vida, ya que las mismas pueden ser positivas o negativas dependiendo de la autopercepción del estudiante del estilo de vida, teniendo claro como este lleva a cabo sus actividades diarias dentro y fuera del ámbito escolar. Los resultados obtenidos muestran que las actividades extracurriculares no tienen impacto en la autopercepción del estudiante en su estilo de vida.

Palabras clave: Actividades extracurriculares, estilo de vida , estudiantes de enfermería.

Abstract: Introduction: Extracurricular activities are those occupations not belonging to the school curriculum, but which influences the person to develop recreational skills and abilities where they can have an impact on the lifestyle of nursing students. Objective: Prove the relationship of extracurricular activities with the lifestyle of nursing students of the Autonomous University of Aguascalientes. Methodology: Descriptive cross-sectional study at the relational level, performed on nursing students with a sample of 221 students obtained by simple random probabilistic sampling without replacement. 


\begin{abstract}
The "FANTASTIC $®$ " questionnaire was used, with 0.789 of Cronbach's Alpha. Results: The average age of the studied population was 20 years, predominantly female with $81.9 \%$. The lifestyle according to student's self-perception was good with $52 \%$, students who do not perform extracurricular activities preponderate with $55.2 \%$. No statistically significant association was found between lifestyle and extracurricular activities. ( $c 2=0.603, p=.896$ ). Conclusion: Extracurricular activities will not always be significant in lifestyle, since they can be positive or negative depending on the student's self-perception in lifestyle, being clear, how it is carried out in daily activities inside and outside the school environment. The results obtained show that extracurricular activities have no impact on the student's selfperception lifestyle.
\end{abstract}

Keywords: Extracurricular activities, lifestyle and nursing students.

\title{
INTRODUCCIÓN
}

La Organización Mundial de la Salud $(\mathrm{OMS})^{1}$ señala que el estilo de vida se refiere a "una forma general de vida basada en la interacción entre las condiciones de vida en un sentido amplio y los patrones individuales de conducta determinados por factores socioculturales y características personales".

El constructo actividad extracurricular se entiende como las actividades complementarias al desarrollo educativo en el salón de clases que lleva a cabo el alumno; generalmente, depende de la institución educativa y es planificada y desarrollada por esta. ${ }^{2}$ Estas actividades extracurriculares deben cumplir con dos premisas: la primera, no ser parte de la currícula del alumno, y, segunda, que forme al alumno trascendiendo la mera socialización. ${ }^{3}$ De manera práctica, las actividades extracurriculares son todos aquellos programas que satisfacen dos condiciones básicas: no son parte del programa curricular de la escuela y tienen cierta estructura no solo para socializar, sino para llegar a una misión o meta. ${ }^{4}$

Los estilos de vida se han estudiado ampliamente en las últimas décadas. Comprenden una serie de hábitos y conductas, tales como alimentación, actividad física, aspectos psicológicos, emocionales y espirituales, incluyendo las relaciones sociales. ${ }^{5}$ Está plenamente demostrado que llevar un estilo de vida saludable favorece el estado salud-enfermedad al disminuir factores de riesgo para enfermar o morir, como: mejor estado físico, nutricional, reducción del estrés, calidad del sueño, administración del tiempo libre, disminución de accidentes, entre muchos otros. ${ }^{6}$

En México, en la actualidad, los nuevos estilos de vida han conllevado el desarrollo de hábitos y costumbres no saludables que incrementan el riesgo de desarrollar principalmente enfermedades crónico-degenerativas, tales como la diabetes mellitus, enfermedades cardiacas, respiratorias y cáncer, entre otras. Lo anterior es resultado de una transición alimentaria, pasando de una dieta rica en frutas, verduras, cereales y leguminosas a una dieta rica en grasas y azúcares refinados. A esto se suma la falta de actividad física, lo que genera sedentarismo en los individuos; además de otros hábitos perjudiciales para la salud como lo 
son el consumo regular de tabaco y alcohol, alteraciones de las horas de sueño e incremento en el estrés. Todo ello da como resultado sobrepeso y obesidad que son, quizás, uno de los principales determinantes de muchas enfermedades no transmisibles. ${ }^{7}$

Así pues, entre los aspectos claves del concepto, se resalta su asociación a conductas de carácter relativamente permanentes relacionadas con la salud del individuo, y que son sensibles de potenciarse o limitarse por las decisiones tomadas a partir de las condiciones del contexto social. ${ }^{8}$

Durante el periodo universitario, los jóvenes se encuentran en un momento crítico de su vida, ya que el desarrollo de sus estilos de vida impactará en su desempeño y su salud a futuro. ${ }^{9}$

Los estudiantes universitarios que desarrollan actividades extracurriculares presentan estilos de vida que, para su percepción, quizá sean adecuados o comunes, desarrollando actividades que tienen impacto importante en su vida cotidiana y en su futuro.

Las enfermedades crónicas no transmisibles son la nueva amenaza que acecha a la población mundial, por lo cual se consideran motivo de preocupación y estudio de los expertos en salud pública. Asimismo, las investigaciones se han centrado en buscar el impacto de los factores biológicos, psicológicos, sociales y culturales en el estilo de vida de las personas y su relación con el mantenimiento de la salud y la aparición de la enfermedad. ${ }^{10}$

La pegunta de investigación planteada fue: ¿Existe relación entre las actividades extracurriculares con el estilo de vida de los estudiantes de enfermería de la Universidad Autónoma de Aguascalientes?

El objetivo general del estudio fue probar la relación de las actividades extracurriculares con los estilos de vida de los estudiantes de enfermería de la Universidad Autónoma de Aguascalientes.

\section{MATERIAL Y MÉTODO}

Se realizó un estudio cuantitativo descriptivo de corte transversal a nivel relacional, en una población de 524 estudiantes de primero a octavo semestre de la Licenciatura en Enfermería de la Universidad Autónoma de Aguascalientes. La investigación se desarrolló con 221 alumnos por medio de marco muestral probabilístico aleatorio simple sin reemplazo. Los alumnos fueron seleccionados por un número aleatorio; al no encontrarse se tomó a la persona siguiente en la lista. El marco muestral lo constituyeron quienes cumplieron los siguientes criterios de inclusión: ser estudiante de la licenciatura, de ambos sexos, de cualquier edad; se excluyeron sujetos que estuvieran en su año de pasantía.

Esta investigación se consideró sin riesgo, de conformidad a lo establecido en la normatividad mexicana. ${ }^{11}$

El instrumento de recolección de datos utilizado fue el cuestionario "FANTASTIC $®$ ", en su versión de cinco opciones de respuesta, ${ }^{12}$ con un alfa de Cronbach del 0.789 , el cual está formado por 24 ítems cerrados que exploran: La relación con la familia y amigos; Actividad física; Nutrición; Consumo de tabaco y otras toxinas; Consumo de alcohol; Horas de sueño, uso de cinturón de seguridad, presencia de estrés; Tipo de personalidad; Pensamientos positivos, 
ansiedad, depresión; Satisfacción con las labores que se desempeñan. Su formato de respuesta es de tipo Likert, de cuatro alternativas con un rango de 0 a 4, con un puntaje total de hasta 100 puntos. Los rangos de calificación que definen el Estilo de Vida (EV) son: hasta 39 puntos = Peligro, 40-59 puntos = Bajo, 60-69 puntos $=$ Regular, 70-84 puntos $=$ Bueno, $85-100$ puntos $=$ Excelente.

La información fue recolectada mediante aplicación individual del cuestionario, se realizó durante cuatro días consecutivos iniciando con el turno matutino, posteriormente el vespertino. Primeramente, se dio la presentación de los investigadores con los estudiantes dando una breve explicación sobre la investigación. Se generó la selección de los estudiantes de manera aleatoria simple, para la aplicación del instrumento. Entrega del consentimiento informado en conjunto con el instrumento para ser contestado. Posteriormente, la recolección de los instrumentos y conteo final sobre la obtención de instrumentos de estudiantes seleccionados.

Para el procesamiento de los datos se vaciaron al programa SPSS $\circledast_{\circledast}$ versión 26, realizando el análisis descriptivo con medidas de tendencia central y dispersión para las variables sociodemográficas y análisis inferencial con la estimación para la proporción poblacional y prueba de correlación para variables categóricas con un nivel de significación $<0.05$.

Dentro de los aspectos éticos y legales, se siguieron los principios establecidos en el Reglamento de la Ley General de Salud en Materia de Investigación para la Salud ${ }^{12}$ y la declaración de Helsinki guardando proporción de ningún riesgo y la protección de su privacidad y confidencialidad.

\section{RESULTADOS}

El estudio contó con 221 participantes; $81.9 \%$ fueron de sexo femenino y $18.1 \%$ de sexo masculino. La edad promedio fue de 20 años, con una desviación estándar de 1.94. De la totalidad de la muestra, el $94.6 \%$ de los estudiantes está soltero, resaltando un 3.2\% que vive en unión libre. Prevaleció el número de estudiantes que no realizan actividades extracurriculares, con un 55.2\%; el restante $44.8 \%$ sí realizaban alguna. El estilo de vida conforme a la autopercepción del estudiante fue de bueno a excelente con un 57.4\%; con tan solo $14 \%$ que tenían un estilo de vida bajo (gráfica 1 ).

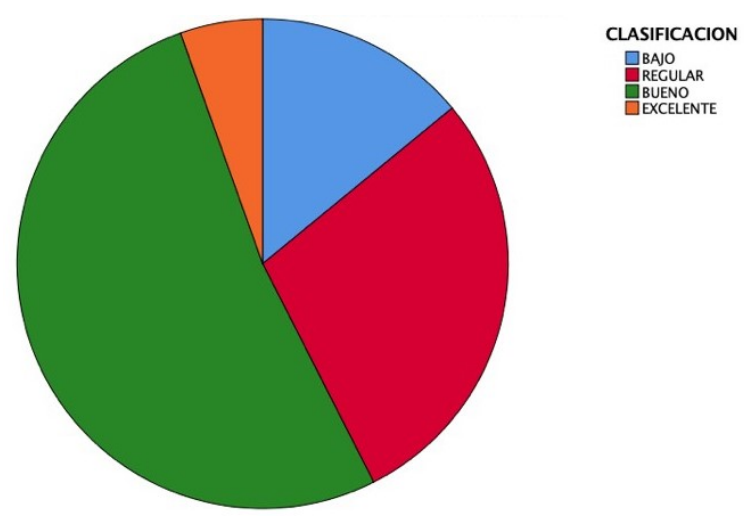

Gráfico 1. Autopercepción del estilo de vida 
De los factores considerados para medir el estilo de vida, el 93.2\% refirió que frecuentemente o casi siempre la comunicación con los demás es honesta, abierta y clara; el 79.2\% afirmó que obtiene el apoyo emocional que necesita; el 43\% aseguró realizar ejercicio activo como correr, andar en bicicleta o caminar rápido tres o más veces a la semana; solo el $14.9 \%$ se relaja y disfruta de su tiempo libre casi a diario, en tanto que el 7.2\% casi nunca lo experimenta; el 53\% consideró llevar una alimentación equilibrada frecuentemente o casi siempre; el 78.3\% desayuna diariamente con una frecuencia de frecuentemente a casi siempre; solo el 33.5\% de los encuestados refirió que rara vez o casi nunca consumieron en exceso azúcar, sal, grasas animales o comida chatarra, aunque fueron pocos los que sí lo hacían casi a diario (3.2\%); en cuanto al peso ideal, el 26.7\% consideró tener un sobrepeso mayor a dos kilogramos; en relación al consumo de tabaco, el 81.9\% no había fumado entre los últimos seis meses y los últimos cinco años, sin embargo todavía un $18.1 \%$ eran fumadores activos; en cuanto al abuso de drogas prescritas y sin prescribir, el 2.8\% manifestó consumirlas de algunas veces a frecuentemente; el consumo de café té o refrescos de cola mantiene un consumo elevado, pues solo el $21.3 \%$ nunca los había consumido; el resultado de beber alcohol y conducir mostró que el $2.7 \%$ lo hace frecuentemente o por lo menos una vez al mes; en relación a las horas de sueño por la noche, el 30.3\% refirió dormir de 7 a 9 horas frecuentemente a casi siempre; en cuanto al uso del cinturón de seguridad, solo el $44.3 \%$ refirió usarlo siempre; el $82.8 \%$ aseguró haber tenido al menos un episodio de estrés importante en el año anterior, el $17.2 \%$ manifestó haber tenido más de cinco de estos episodios, lo que es preocupante para personas que todavía son estudiantes; el rubro anterior se corrobora con los episodios de sensación de urgencia o impaciencia, de los cuales solo el $14.5 \%$ manifestó casi nunca tenerlos, mientras que el 3.2\% refirió tenerlos casi siempre; el 46.2\% consideró casi nunca tener competitividad y agresividad; un porcentaje similar (45.7\%) casi nunca tenían sentimientos de ira y hostilidad; el $38 \%$ casi siempre pensaban de manera positiva; sin embargo, solo el $13.6 \%$ casi nunca sufrió de ansiedad y preocupación; otro factor importante fue la percepción de estar deprimidos, solamente el $39.4 \%$ afirmó que casi nunca lo sentía; el 74.2\% aseguró sentirse de frecuentemente a casi siempre satisfecho con el trabajo o las labores que desempeña; finalmente, el $60.2 \%$ refirió tener casi siempre buenas relaciones con quienes le rodean. Se realizó el análisis estadístico mediante la prueba $X^{2}$ para determinar si existía asociación entre las variables actividad extracurricular y auto percepción del estilo de vida (tabla 1).

Tabla 1. Clasificación de la percepción del estilo de vida y actividad extracurricular

\begin{tabular}{lclllll}
\hline & \multicolumn{7}{c}{ CLASIFICACIÓN } \\
& Bajo Regular & Bueno Excelente & Total \\
\hline Actividad & Sí & 15 & 26 & 53 & 5 & 99 \\
extracurricular & NO & 16 & 37 & 62 & 7 & 122 \\
\hline Total & & 31 & 63 & 115 & 12 & 221 \\
\hline
\end{tabular}

El resultado mostró que no existe asociación entre estas variables categóricas $\left(X^{2}=0.603, p=.896\right)($ tabla 2$)$. 
Tabla 2. Pruebas de Chi-cuadrado

\begin{tabular}{llll}
\hline & Valor & df & $\begin{array}{c}\text { Significación } \\
\text { asintótica } \\
\text { (bilateral) }\end{array}$ \\
\hline $\begin{array}{l}\text { Chi-Cuadrado de } \\
\begin{array}{l}\text { Pearson } \\
\text { Razón de } \\
\text { verosimilitud }\end{array}\end{array}$ & $0.603 a$ & 3 & 0.896 \\
$\begin{array}{l}\text { Asociación lineal por } \\
\text { lineal }\end{array}$ & 0.004 & 1 & 0.949 \\
$\begin{array}{l}\text { N de casos válidos } \\
\text { a: 0 casillas (0.0\%) han esperado un recuento menor } \\
\text { que 5. El recuento mínimo esperado es 5.38. }\end{array}$
\end{tabular}

\section{DISCUSIÓN}

Recordemos que las actividades extracurriculares son aquellas que no entran en el ámbito académico; por lo cual, hablando en estancia universitaria, dichas actividades podrían considerarse que tienen relación en cuanto al estilo de vida que lleva cada universitario.

Los hallazgos de este estudio concuerdan con las encontradas por PozónLópez, ${ }^{13}$ quien probó que las actividades extracurriculares son de suma importancia en el desarrollo del estudiante universitario, pues contribuyen a la formación integral de los jóvenes y a generar una vida universitaria más atractiva. No obstante, dichas actividades deben ser solo un complemento del plan de estudios. Este autor demostró una fuerte apatía de los estudiantes para llevar a cabo actividades extracurriculares, lo que se vincula en sentido negativo con el desarrollo profesional de los jóvenes.

Desde una visión psicopedagógica, el estudio de Francisca Alcántara-Paisán et $a l{ }^{4}$ demostró que las actividades extracurriculares desarrolladas por estudiantes de las ciencias de la salud (entre ellas enfermería), impactan positivamente en su desempeño profesional futuro. Específicamente, estas actividades promueven la participación colaborativa, el liderazgo activo, la elevación de la autoestima y sus metas profesionales. Además, favorecen las relaciones interpersonales incrementando el compromiso moral consigo mismos y con los demás. Quizá uno de los elementos más importante es que les permite desarrollar "aprendizajes significativos" que les ayudarán a planear su futuro y a ser resilientes antes las adversidades en su vida profesional. Sus resultados mostraron que el estilo de vida conforme a la autopercepción del estudiante fue bueno, con un 52\%, independientemente de si realizan actividad extracurricular (44.8\%) o no realizan actividad extracurricular (55.2\%); encontrándose que tan solo en el 14\% se identificó un estilo de vida bajo. Esto se compara con los hallazgos de MontoyaBecerril, ${ }^{14}$ quien trabajó con 169 estudiantes de sexto de bachillerato de la ciudad de Morelia, Michoacán, donde encontró que la práctica de actividades artísticas extracurriculares sí favorece el desempeño académico escolar, reflejado mayormente en sus calificaciones. 
La investigación de Herrada-Lemus et al. ${ }^{15}$ asevera que, en su mayoría (46\%), los estudiantes consideraron su alimentación como buena, de la misma manera en este estudio se encontró que llevan una alimentación equilibrada (37.4\%) a pesar del consumo excesivo de grasas, sal y comida chatarra. No obstante encontrarse en un nivel educativo superior, los estudiantes universitarios han manifestado que la ingesta de alcohol y tabaco son productos que se consumen frecuentemente, pues se observó un porcentaje considerable de consumo de alcohol, siendo solo en el $5.9 \%$ de manera ocasional. Por otro lado, se encontró que el consumo de tabaco representó $15.4 \%$ de 1 a 10 veces por semana, en comparación con AdasSaliba, ${ }^{16}$ quien en su estudio encontró un $80 \%$ de consumo de alcohol.

Esta investigación aporta resultados similares a los encontrados por Morales M. et al., ${ }^{17}$ ya que sus datos reflejan que la gran mayoría de los participantes no consume tabaco (80.97\%); en tanto que, en nuestra investigación, el 81.9\% no había fumado por lo menos en los últimos seis meses.

Nuestros resultados concuerdan con los de Lara et al., ${ }^{18}$ en que los estudiantes necesitan mejorar aspectos correspondientes a actividad física, alimentación poco saludable, fumar, consumo de bebidas alcohólicas, hábitos de sueño y manejo del estrés.

También se ha descrito que los hábitos adquiridos y desarrollados en la edad infantil, extendiéndolos durante la adolescencia, persisten en la edad adulta, como lo señalan Montero-Bravo ${ }^{19}$ y Rizo-Baeza. ${ }^{20}$

Independientemente de los resultados obtenidos, en su mayoría, los alumnos siguen mostrando que, de acuerdo a su percepción, consideran tener un estilo de vida bueno; pero aún es necesario orientar a los alumnos en cuanto alimentación, estilos de vida o aquellas situaciones que los afecten en su rendimiento académico, repercutiendo sobre todo en su salud.

\section{CONCLUSIÓN}

Los resultados obtenidos muestran que las actividades extracurriculares no tienen relación con el estilo de vida, según la autopercepción del estudiante, ya que la mayoría considera tener un buen estilo de vida independientemente de si realizan o no actividades extracurriculares. Es necesario desarrollar estrategias de involucramiento en actividades extracurriculares por parte de los alumnos de enfermería ligados a estilos de vida saludables que repercutan en la vida futura del profesionista, procurando el empoderamiento mediante aprendizajes significativos que perduren a lo largo de su vida personal y profesional.

\section{Referencias}

1. Cerón-Souza C. Editorial. Universidad y Salud. 2012 [Consultado 27 Jun 2021 ];14(2):115-115. Disponible en: http://www.scielo.org.co/scielo.php? script=sci_arttext\&pid=S0124-71072012000200001\&lng=en\&nrm=iso\&tlng=es

2. Moriana-Elvira JA, Alós-Cívico F, Alcalá-Cabrera R, Pino-Osuna MJ, HerruzoCabrera J, Ruiz-Olivares R. Actividades extraescolares y rendimiento académico en alumnos de Educación Secundaria. Electronic Journal of Research in 
Educational Psychology [Internet]. 2006 [Consultado 27 Jun 2021]; 4(1):35-46. Disponible en: http://www.redalyc.org/articulo.oa?id=293123488002

3. Brown R. Extracurricular Activity: How does participation encourage positive ... - [Internet]. University of Nevada. Cooperative Extension. [Consultado 27 Jun 2021]. p. indefinida. Disponible en: https://slidelegend.com/extracurricular-activity-how-does-participationencourage-positive-_59e840421723dde34805221e.html

4. Alcántara-Paisán F de la C, Alcántara-Paisán A, Vara-Moya EJ, Fimia-Duarte R. Impacto de actividades extracurriculares de formación vocacional y orientación profesional en estudiantes de ciencias médicas [Internet]. En línea. 2017 [Consultado 27 Jun 2021]. Disponible en: http://scielo.sld.cu/scielo.php? script $=$ sci_abstract\&pid $=$ S2077-28742017000200010

5. Cedillo-Ramírez L, Correa-López LE, Vela-Ruiz JM, Pérez-Acuña LM, LoayzaCastro JA, Cabello-Vela CS, et al. Estilos de vida de estudiantes universitarios de ciencias de la salud. Revista de la Facultad de Medicina Humana. 20 Mayo 2016 20;16(2).

6. Jiménez-Diez O, Ojeda-López RN. Estudiantes universitarios y el estilo de vida [Internet]. Revista Iberoamericana de Producción Académica y Gestión Educativa. 2017 [Consultado 27 Jun 2021]. Disponible en: https:// www.pag.org.mx/index.php/PAG/article/view/723/1009

7. Botello-Márquez M, Villanueva-Lugo NE, Ruiz-Vargas NV, Gallegos-Torres RM. Alimentación y estilos de vida saludables percibidos en estudiantes universitarios del área de la salud, de tres licenciaturas. Lux Médica [Internet]. Sep 2018 [Consultado 27 Jun 2021]; 39(13):55-62. Disponible en: https:// revistas.uaa.mx/index.php/luxmedica/article/view/1334/1279

8. Muñoz-Argel M, Uribe-Urzola A. Estilos de vida en estudiantes universitarios. Psicogente [Internet]. 1 Jul 2013 [Consultado 27 Jun 2021]; 16(30):356-67. Disponible en: https://doaj.org/article/096b3b50af8a4684b00cef3f198384b9

9. Gómez-Cruz Z, Landeros-Ramírez P, Romero-Velarde E, Troyo-Sanromán R. Estilos de vida y riesgos para la salud en una población universitaria. Revista Salud Pública y Nutrición [Internet]. 10 Jul 2016 [Consultado 27 Jun 2021]; 15(2):15-21. Disponible en: https://respyn.uanl.mx/index.php/respyn/article/view/11

10. Suescún-Carrero SH, Sandoval-Cuellar C, Hernández-Piratoba FH, AraqueSepúlveda ID, Fagua-Pacavita LH, Bernal-Orduz F, et al. Lifestyle in students of a university in Boyacá, Colombia. Revista Facultad de Medicina [Internet]. 1 Abr 2017 [Consultado 27 Jun 2021]; 65(2):227-31. Disponible en: https:// revistas.unal.edu.co/index.php/revfacmed/article/view/58640

11. Cámara de Diputados del H. Congreso de la Unión. Reglamento de la Ley General de Salud en materia de Investigación para la Salud. Diario Oficial de la Federación. Última Reforma Publicada 02-04-2014. p. 1-31.

12. López-Carmona J, Rodríguez-Moctezuma R, Munguía-Miranda $C$, HernándezSantiago J, Casas de la Torre E. Validez y fiabilidad del instrumento "FANTASTIC" para medir el estilo de vida en pacientes mexicanos con hipertensión arterial. Atención primaria. 15 Nov 2000; 26(8):542-9.

13. Pozón-López JR. Los estudiantes universitarios ante las actividades extracurriculares. Universidad Anáhuac México Sur. 20 Ene 2014; (13):137-50.

14. Montoya-Becerril G, Oropeza-Tena R, Ávalos-Latorre ML. Rendimiento académico y prácticas artísticas extracurriculares en estudiantes de bachillerato. Revista Electrónica de Investigación Educativa Versión online [Internet]. 15 Abr 2019 [Consultado 27 Jun 
2021]; 21(1). Disponible en: http://www.scielo.org.mx/scielo.php? script=sci_arttext\&pid=S1607-40412019000100113\&lng=es\&nrm=iso\&tlng=es

15. Herrada-Lemus JV, Casillas-Bonilla AL, García-Magdaleno D, Ramírez-Martínez MM, Reyes-Ruiz Esparza II, Martín del Campo-Cervantes J. Hábitos alimenticios en estudiantes de Nutrición de la Universidad Autónoma de Aguascalientes en el periodo enero- junio de 2015. Lux Médica [Internet]. 2016 [Consultado 27 Jun 2021]; 33(2):3-8. Disponible en: https://revistas.uaa.mx/index.php/luxmedica/ article/view/720/697

16. Adas-Saliba T, Santos-de Sandre A, Adas-Saliba Moimaz S, Adas-Saliba Garbin C. Perfil de estilo de vida individual de estudiantes de primer año de odontología de la Universidad de Aracatuba, Brasil - 2015. Universidad y Salud Facultad de Odontología de Aracatuba-UNESP Brasil [Internet]. 2017 [Consultado 27 Jun 2021]; 19(2):258-66. Disponible en: https://revistas.udenar.edu.co/index.php/ usalud/article/view/3016/pdf

17. Morales M, Gómez V, García C, Chaparro-Díaz L, Carreño-Moreno S. Estilos de vida saludable en estudiantes de enfermería del Estado de México. Rev Colomb Enferm . Abr 2018; 16(13):14-24.

18. Lara Y, Quiroga C, Jaramillo A, Bermeo M. Estilo de vida de estudiantes en primer semestre de odontología de una universidad privada, Cali 2016. Revista Odontológica Mexicana. 2018; 22(3):144-9.

19. Montero-Bravo A, Úbeda-Martín N, García-González A. Evaluación de los hábitos alimentarios de una población de estudiantes universitarios en relación con sus conocimientos nutricionales. Nutrición Hospitalaria Versión online [Internet]. 2006 [Consultado 27 Jun 2021]; 21(4). Disponible en: https://scielo.isciii.es/ scielo.php?script $=$ sci_arttext\&pid $=$ S0212-16112006000700004

20. Rizo-Baeza MM, González-Brauer NG, Cortés E. Calidad de la dieta y estilos de vida en estudiantes de Ciencias de la Salud. Nutrición Hospitalaria Versión online [Internet]. 1 Ene 2014 [Consultado 27 Jun 2021];29(1):153-7. Disponible en: https://scielo.isciii.es/scielo.php? script $=$ sci_arttext\&pid=S0212-16112014000100020\&lng=es\&nrm=iso\&tlng=es 